Journal of Economics and Behavioral Studies

Vol. 5, No. 8, pp. 522-534, Aug 2013 (ISSN: 2220-6140)

\title{
The Impact of Husband's Perception and Value towards Family Purchase Decision Patterns
}

\author{
${ }^{*}$ Christian Wibisono, Mustika Sufiati Purwanegara \\ Institute of Technology Bandung, School of Business and Management, Indonesia \\ *christian.w@sbm-itb.ac.id
}

\begin{abstract}
Changes are occurring in society's norm structure. One of these changes is the shifting of women roles. More wives are working outside home and acting as a source of income, event becoming chief wage earner in the family. Many past researches have used demographic and family structure as predictor of family purchase decision patterns. This study will figure out the impact of husbands' perception and value to the usage of wife's income for family expenditure. Total 112 husbands in Jakarta and Bandung participated in this survey. This study used questionnaire to gather data. Questions to measure perception and norm of husbands were derived from qualitative study, a preliminary interview with 20 husbands in Jakarta and Bandung Indonesia. Schwartz Value Scale was used to measure husband's value structure. Findings indicate that husband perception and value have significant impact on usage of wife's income for family expenditure. This research is useful to fill the research gap in family purchase decision theory. For marketers and business practitioners the results will enhance their comprehension in segmenting, selecting more effective target and creating more creative communication strategy.
\end{abstract}

Keywords: Working wife, Perception, Norm, Schwartz Value, Household Purchase Decision, Family Decision Making Process

\section{Introduction}

Family is an important unit of analysis in consumer decision-making field (Xia et al., 2006), but the vast interest in family as unit of analysis in research has not happened only until recently (So and Yao, 2006). Family is a main reference group when individual family member is making purchase decision (Kotler, 2002). Recent researches have shown that decision to consume most goods and services by individual are made in the family rather than individually (Makgosa, 2007). Many researchers are aware of this important role that family plays in the field of consumer behavior. Accordingly, many researches in the field of consumer behavior were conducted with family as the main unit of analysis in the research, especially in understanding the way families make purchase decision. Many past studies have used family structures and demographic as predictor of family decision-making pattern. Other studies have examined the different roles of husband and wife in family decision making when making decision to purchase goods and services together. Xia et al (2006) stated the role of husband and wife when making purchase decision is different from time to time in regard to the product category being considered, the amount of resources possessed by each spouses, and stage of decision-making process. According to Stafford et al. (2011), Sex Role Orientation (SRO) has strong influence on degree of influence each spouses has on family purchase decision-making (Scanzoni 1977; Qualls 1987; Schaninger, Buss and Grover, 1982). Another determinant of degree of influence of husband and wife in family decision-making were education (Blood and Wolfe, 1960; Rosen and Granbois, 1983; Spiro, 1983), wife employment status (Spiro, 1983), household income (Blood and Wolfe, 1960; Filiatrault and Ritchie, 1980; Spiro, 1983), and presence of Children (Filiatrault and Ritchie, 1980; Spiro, 1983). We can see that many researches focused on demographic and family structure in predicting family decision-making patterns and most of them use western families as the unit of analysis. Very few evidence of research in family decision making that use values as predictor of family decision-making patterns. It is important to understand that even though the unit of analysis is family, the interaction and behavior among individuals within the families are highly affected by the value structure of family member.

Importance of understanding the pattern of family decision making should not only be the interests of researchers in western societies, because phenomena of family as buying unit is happening everywhere 
throughout the world, not only in western societies (Harcar et al., 2005). The importance to renew our understanding of family decision-making is also increasing because value changes have occurred in societies. This shift in value has made wife to posses more and more influence in family decision making which in turn will change the structure of family and role of husband and wife. It will significantly affect the way husband and wife made purchase decision in household. This changes in value are due to more women are working outside home, pursuing career in office, and receiving higher and higher education now compare to 10 or 20 years later (Lee, 2002). Most research investigated the impact of changing role of women to family decisionmaking process. However, not many researchers have investigate the impact of this changing role of women and the changes occurred in family decision making pattern that it causes from the husbands' perspective. For example, how the husband react to this changes in family structure and values and how he sees the decision making process in family nowadays (So et al., 2006). Therefore, the purpose of this paper is to investigate the effect of individual values, norms, and perception on family decision-making process from the husband's perspectives. Geographically, the research is conducted in Jakarta and Bandung (Indonesia) where there is still very few-if not- no researches about family decision-making process.

\section{Literature Review}

Hypotheses Development: The study consisted of two stages: qualitative and quantitative method. Qualitative method was applied at the earlier part of the study to identify factors that are regarded as important factor in affecting husband's perception about their working wife and the usage of wife income for family expenditure.

Husband's Schwartz Value Structure: Value has been a centre of study in the field of consumer behavior for a very long time. Values are believed to be significant influencers of individual attitude towards something, which in turn will affect individual behavior. According to Rokeach, values are "enduring beliefs that a specific mode of conduct is personally or socially more preferable to an opposite or converse mode of conduct or endstate of existence" (Rokeach, 1973). According to the Value - Attitude - Behavior Model, individual attitudes and behaviors can be traced back to his or her values (Vanvara, 2009). It is said that there is no direct relationship between value and behavior, but there is a mediating role that attitudes plays between value and behavior (Vanvara, 2009). This means, values can be used as root predictor of individual's behavior, although there are no direct relationship between values and behavior. Schwartz believed that values are well defined and perceived the same across cultures, therefore it is possible for researcher to measure the relationship of values with other variables such as beliefs, attitudes, and behavior. Schwartz value scale has been used to predict behavior of individual towards environmental issues. Besides, it is also has been used to predict consumer behavior in buying products (Vanvara, 2009).

Therefore the hypotheses are:

Hypotheses 1a (H1a): Husband's personal values positively affect husband's norm belief towards his working wife.

Hypotheses 1b (H1b): Husband's personal values positively affect husband's perception towards his working wife.

Hypotheses 1c (H1c): Husband's personal values positively affect usage of wife's income for family expenditure.

Husband's Social Norm: According to Kandori (1992) social norm consist of two factors: expected behavior and sanctions. It is not written, but member of society usually will obey it. The social norm will guide the behavior of individual in the society. It is a code of conduct that existed in a society in which its member should act or behave accordingly in a given situation. The group in which social norms prevail can be a family, a peer group, an organization, or even a society (Fehr and Fischbacher, 2004). Fehr and Fischbacher (2004) believe that norms within society will guide behavior of people in doing something. If the required behavior is in accordance to a person individual goal, he or she will obey the norm voluntarily. However, if it conflicted with the person's personal goal, then he or she will obey the norm because afraid of social sanction. The work of Fehr and Fischbacher (2004) goes in line with the work of Lapinski and Rimal (2005) who stated that 
human behavior is guided by the perceptions about the popularity of the behavior. Norm of society will affect the perceived popularity of the behavior, which in turn will affect behavior of individual within the society. Therefore the hypothesis is:

Hypothesis 2 (H2): Husband's norm about wife who is working outside home will positively affect husband's perception towards his working wife.

Husband's Perception towards Working Wife: Changes in societies have given opportunities for women to work outside home and pursue career, receive higher education, and make women able to contribute to create income for household, role that were traditionally performed by husbands (Qualls, 1987: Lee and Beatty, 2002). With that, the strict division of role of husbands and wives who originally have the role as main source of income and wives who have original role to housekeeping and childcare are fading (Ndubisi and Koo, 2006). One of the affecting factors of spousal influence in family decision-making is spousal resources (Yang et al., 2006). With wife also contributing to family income, husband will now have to step down to more equal role with his working wife in deciding purchase of goods and services. It is interesting to see how husband react to this changes of role in family, for example, how husband react to new family relationship structure and to new power distribution in decision making (So and Yau, 2006). Gianopulos (1957) stated that husbands' perception towards wife's employment is one of the important factors in determining level of conflict in family decision-making process. We can infer that husband's perception will affect his attitude towards his wife's employment status. Therefore the hypotheses are:

Hypotheses 3 (H3): Husband's perception towards his working wife will positively affect usage of wife income for family expenditure.

Resource Allocation in Family Decision Making: Family decision-making is different from individual decision-making. Family decision-making involved at least two of family members directly or indirectly making decision towards purchasing goods or services (Harcar et al., 2005). This paper will adopt these definitions of family and family decision-making. As earlier part of the paper stated, family is an important unit of analysis in consumer behavior field. Many past researches have tried to differentiate family decisionmaking type as husband dominant, wife dominant, or joint (Ndubisi and Koo, 2006; Martinez and Polo, 1994; Yavas, Babakus and Delener, 1994 in Makgosa, 2007). From many past researches, we can see that perception of husband towards his wife and husbands' norm and value structure has not been used as predictors of family decision-making. Few studies have been conducted in examining usage of wife's income for family expenditure. Therefore, this paper will propose the model as can be seen in Figure 1.

Figure 1: Conceptual Framework

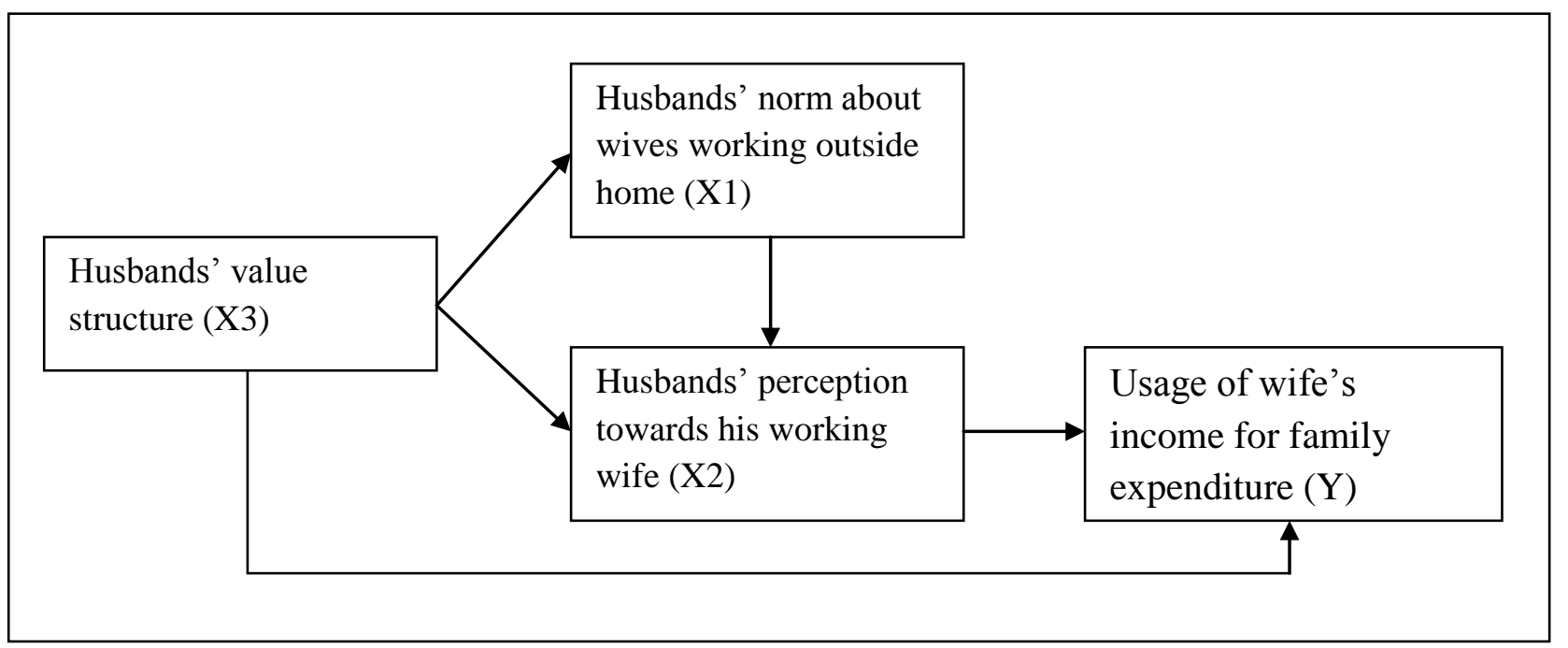




\section{Methodology}

Sample and Data Collection: The sample consisted of 112 husbands. Most of them currently live in Jakarta and Bandung. Table 1 shows demographic profiles of the respondents.

Table 1: Demography Characteristics of Sample

\begin{tabular}{|c|c|c|c|}
\hline Variables & Categories & Frequency & Percentage \\
\hline \multirow[t]{6}{*}{ Age } & $21-27$ у.о & 8 & 7.14 \\
\hline & $28-35$ у.о & 33 & 29.5 \\
\hline & $36-43$ у.о & 28 & 25.0 \\
\hline & 44-51 у.о & 14 & 12.5 \\
\hline & $52-59$ у.о & 26 & 23.2 \\
\hline & $>59$ y.o & 3 & 2.7 \\
\hline \multirow[t]{5}{*}{ Years of Marriage } & $0-5$ years & 38 & 33.9 \\
\hline & $6-10$ years & 20 & 17.9 \\
\hline & $11-15$ years & 12 & 10.7 \\
\hline & $16-20$ years & 14 & 12.5 \\
\hline & $>20$ years & 28 & 25.0 \\
\hline \multirow[t]{4}{*}{ No. of children } & 0 & 17 & 15.2 \\
\hline & $1-2$ & 79 & 70.5 \\
\hline & $3-4$ & 12 & 10.7 \\
\hline & $>4$ & 4 & 3.6 \\
\hline \multirow[t]{3}{*}{ Education } & Junior High & 0 & 0 \\
\hline & Senior High & 10 & 8.9 \\
\hline & Bachelor & 102 & 91.1 \\
\hline \multirow[t]{2}{*}{ Living Place } & Jakarta & 57 & 50.9 \\
\hline & Outside Jakarta & 55 & 49.1 \\
\hline \multirow[t]{2}{*}{ Origin } & Jakarta & 49 & 43.8 \\
\hline & Outside Jakarta & 63 & 56.3 \\
\hline \multirow[t]{3}{*}{ Race } & Javanese & 20 & 17.9 \\
\hline & Sundanese & 14 & 12.5 \\
\hline & Chinese & 56 & 50.0 \\
\hline \multirow[t]{7}{*}{ Religion } & Others & 22 & 19.6 \\
\hline & Christian & 25 & 22.3 \\
\hline & Catholic & 57 & 50.9 \\
\hline & Moslem & 28 & 25.0 \\
\hline & Budha & 2 & 1.8 \\
\hline & Hindu & 0 & 0 \\
\hline & Others & 0 & 0 \\
\hline
\end{tabular}

Paper and online questionnaires were administered to collect data for this research. The online questionnaires were distributed through social media, mailing list, and personal email. Offline questionnaires were distributed personally. Questionnaires were self-administered and convenience sampling was used because of time constraint.

Measures: The study was conducted in two steps. The first step was literature review and in-depth interview to find factors that will affect husband's perception and norm towards his working wife. Based on the literature review we have conducted, value, norm, and perception is attitude predictor (Vanvara, 2009; 
McCarty and Shrum, 1994; Gianopulos, 1957). Questionnaires were used to measure each variable. The questionnaire has 4 parts. The first part measures husbands' norm about wives working outside home (X1), the second part measures husbands' perception if wives earn more than him (X2), the third part measures husbands' value structure (X3), and the last part measures usage of wife's income for family expenditure (Y). Questions to measure X1, X2 and Y were derived from preliminary studies and in-depth interview with around twenty husbands who live in Jakarta and Bandung. The in-depth interview helps us to identify what factors considered as important by husbands in perceiving their working wife, what norms do they have about their working wife, and what is the factor that influence their willingness to use wife's income for family expenditure. Meanwhile husband's value structure was measured by adapted scale from Schwartz value scale. Regression was used to measure amount of influence among variables. Validity and reliability analysis was also conducted in order to examine validity and reliability of the questions used to measure the variables.

\section{Results and Discussion}

Measure of reliability: Reliability tests was done to measure questions measuring the following variables: husbands' norm about wives working outside home (X1), husbands' perception if wives earn more than him (X2), husbands' value structure (X3), and usage of wife's income for family expenditure (Y). Table 2 will show the results of the reliability analysis.

Table 2: Reliability analysis result

\begin{tabular}{lll}
\hline Variable & Cronbach's Alpha & $\begin{array}{l}\text { Cronbach's } \\
\text { based } \\
\text { standardized items }\end{array}$ \\
\hline $\begin{array}{l}\text { X1(Husband's norm about wife's working outside of } \\
\text { home) }\end{array}$ & 0.825 & 0.834 \\
X2 (Husband's perception towards his working wife) & 0.824 & 0.886 \\
X3 (Husband's value belief) & 0.888 & 0.909 \\
Y (Usage of wife's income for family expenditure) & 0.879 & 0.881 \\
\hline
\end{tabular}

Cronbach's alpha values can be seen in Table 2, which reveal that all items shows good internal consistency reliability for the scale with this sample. According to Pallant (2011), values above 0.7 are acceptable, but values above 0.8 are preferable.

Validity using factor analysis: Because indicators to measure variables were not derived from theory but from in-depth interview, therefore factor analysis was used to verify the grouping of indicator representing the variable. Factor analysis also used to verify the validity of the construct. Factor analysis conducted using principal component extraction method with varimax rotation, Eigen value greater than 1 , and extracted by factor loading greater than 0.55 for a sample size of 100 respondents (Hair et al, 2010). The results of the factor analysis is shown in Table 3.

According to Heir et al. (2010), a KMO value of less than 0.50 is not acceptable. From the Table 3, we can see that all item of indicator husband's norm, husband's perception, husband's value, and usage of wife's income for family expenditure did not have KMO value below 0.5. This means that the developed scale were adequate. Factor loading of each item must not be less than 0.45 (Hair et al., 2010). Table 4 shows the resultant factors, naming of factors, and items constructing that factor from the result of factor analysis. 
Table 3: Result of factor analysis

\begin{tabular}{|c|c|c|c|c|c|}
\hline Variable & KMO & $\begin{array}{l}\text { Bartlett's test } \\
\text { of sphericity }\end{array}$ & Sig. & $\begin{array}{l}\text { Created } \\
\text { Factors }\end{array}$ & $\begin{array}{l}\text { Total } \\
\text { Explained } \\
\text { Variance }(\%)\end{array}$ \\
\hline $\begin{array}{l}\text { Husbands' } \\
\text { norm about } \\
\text { wives working } \\
\text { outside home } \\
\text { (X1) }\end{array}$ & 0.715 & 847.220 & .000 & 3 & $\begin{array}{l}41.038 \\
20.026 \\
15.147\end{array}$ \\
\hline $\begin{array}{l}\text { Husbands' } \\
\text { perception } \\
\text { towards his } \\
\text { working } \\
\text { wife(X2) }\end{array}$ & 0.685 & 570.446 & .000 & 2 & $\begin{array}{l}69.040 \\
22.277\end{array}$ \\
\hline $\begin{array}{l}\text { Husbands' } \\
\text { value beliefs } \\
\text { (X3) }\end{array}$ & 0.632 & 1899.047 & .000 & 4 & $\begin{array}{l}42.391 \\
15.572 \\
8.325 \\
6.096\end{array}$ \\
\hline $\begin{array}{l}\text { Usage of wife's } \\
\text { income for } \\
\text { family } \\
\text { expenditure } \\
\text { (Y) }\end{array}$ & 0.615 & 305.940 & .000 & 1 & 100 \\
\hline
\end{tabular}

Table 4: Resultant factors, naming of factors, factor loading, and items constructing factor

\begin{tabular}{|c|c|c|c|c|}
\hline Variable & Sub Variable & Factor Name & $\begin{array}{l}\text { Items Constructing } \\
\text { Factor }\end{array}$ & $\begin{array}{l}\text { Factor } \\
\text { Loading }\end{array}$ \\
\hline \multirow{11}{*}{$\begin{array}{l}\text { Husband's } \\
\text { norm about } \\
\text { wives } \\
\text { working } \\
\text { outside } \\
\text { home }\end{array}$} & \multirow[t]{4}{*}{$\begin{array}{l}\text { Working Condition } \\
\text { Norm }\end{array}$} & $\begin{array}{l}\text { 1. Husband's norm about wife's } \\
\text { working condition }\end{array}$ & $\begin{array}{l}\text { Wife's level of } \\
\text { tiredness at work }\end{array}$ & 0.690 \\
\hline & & & $\begin{array}{l}\text { Wife's level of work } \\
\text { stress }\end{array}$ & 0.757 \\
\hline & & & Wife's working & 0.756 \\
\hline & & & environment safety & \\
\hline & \multirow{3}{*}{$\begin{array}{l}\text { Household } \\
\text { Norm }\end{array}$} & \multirow{3}{*}{$\begin{array}{l}\text { 2. Husband's } \\
\text { household duty }\end{array}$} & Child caring & -0.373 \\
\hline & & & Home cleaning & -0.640 \\
\hline & & & Cooking & -0.438 \\
\hline & \multirow{4}{*}{$\begin{array}{l}\text { Wife's } \\
\text { Responsibility } \\
\text { Norm }\end{array}$} & \multirow{4}{*}{$\begin{array}{l}\text { 3. Husband's } \\
\text { responsibility }\end{array}$} & Child education & 0.450 \\
\hline & & & Respect to husband & 0.507 \\
\hline & & & $\begin{array}{l}\text { as head of } \\
\text { household }\end{array}$ & \\
\hline & & & $\begin{array}{l}\text { Respect to husband } \\
\text { in public }\end{array}$ & 0.353 \\
\hline \multirow{5}{*}{$\begin{array}{l}\text { Husband's } \\
\text { perception } \\
\text { towards his } \\
\text { working wife }\end{array}$} & \multirow[t]{3}{*}{ Wife's Respect } & $\begin{array}{l}\text { 1. Husband's perception towards } \\
\text { wife's respect }\end{array}$ & $\begin{array}{l}\text { Wife listen to } \\
\text { husband's opinion }\end{array}$ & 0.844 \\
\hline & & & Wife serve husband & 0.886 \\
\hline & & & Wife obey husband & 0.905 \\
\hline & \multirow[t]{2}{*}{$\begin{array}{l}\text { Maintain } \\
\text { Husband's Prestige }\end{array}$} & $\begin{array}{l}\text { 2. Husband's perception towards } \\
\text { how well wife maintain husband's }\end{array}$ & $\begin{array}{l}\text { Maintain prestige in } \\
\text { public }\end{array}$ & 0.652 \\
\hline & & prestige & $\begin{array}{l}\text { Maintain prestige in } \\
\text { front of the children }\end{array}$ & 0.570 \\
\hline \multirow{2}{*}{$\begin{array}{l}\text { Husband's } \\
\text { value belief }\end{array}$} & \multirow{2}{*}{$\begin{array}{l}\text { Gender } \\
\text { value }\end{array}$} & \multirow{2}{*}{$\begin{array}{l}\text { 1. Husbands } \\
\text { equality value }\end{array}$} & Excitement & 0.768 \\
\hline & & & Warm & 0.648 \\
\hline
\end{tabular}




\begin{tabular}{|c|c|c|c|c|}
\hline & & & Self respect & 0.646 \\
\hline & & & Universalism & 0.715 \\
\hline & & & Tradition & 0.709 \\
\hline & & & Conformity & 0.734 \\
\hline & & & Benevolence & 0.771 \\
\hline & Achievement value & $\begin{array}{l}\text { 2. Husbands who needs to achieve } \\
\text { personal success through } \\
\text { demonstrating competence } \\
\text { according to social standards }\end{array}$ & Achievement & 0.457 \\
\hline & $\begin{array}{l}\text { Accomplishment } \\
\text { value }\end{array}$ & $\begin{array}{l}\text { 3. Husbands who needs to succeed at } \\
\text { what they want to do }\end{array}$ & Accomplishment & 0.457 \\
\hline & Respected value & $\begin{array}{l}\text { 4. Husbands who needs to be } \\
\text { admired by others and to receive } \\
\text { recognition }\end{array}$ & Respected & 0.649 \\
\hline $\begin{array}{l}\text { Usage of } \\
\text { wife's }\end{array}$ & $\begin{array}{l}\text { Usage of Wife's } \\
\text { Income for Family }\end{array}$ & $\begin{array}{l}\text { 1. Using wife's income for family } \\
\text { expenditure }\end{array}$ & $\begin{array}{l}\text { How good is it to use } \\
\text { wife's income }\end{array}$ & 0.787 \\
\hline $\begin{array}{l}\text { income for } \\
\text { family }\end{array}$ & Expenditure & & $\begin{array}{l}\text { Amount of wife's } \\
\text { income used }\end{array}$ & 0.964 \\
\hline expenditure & & & $\begin{array}{l}\text { Frequency of using } \\
\text { wife's income }\end{array}$ & 0.943 \\
\hline
\end{tabular}

Model forming and Regression analysis: We conducted regression analysis to find Beta coefficient (B) to measure the effect between husband's value structure, husband's perception, and husband's norm (independent variables) to usage of wife's income for family expenditure (dependent variable). From the results, not all variables have significant effect towards each other. Table 5 shows the complete results of regression analysis that we conducted.

Table 5: Results of regression analysis

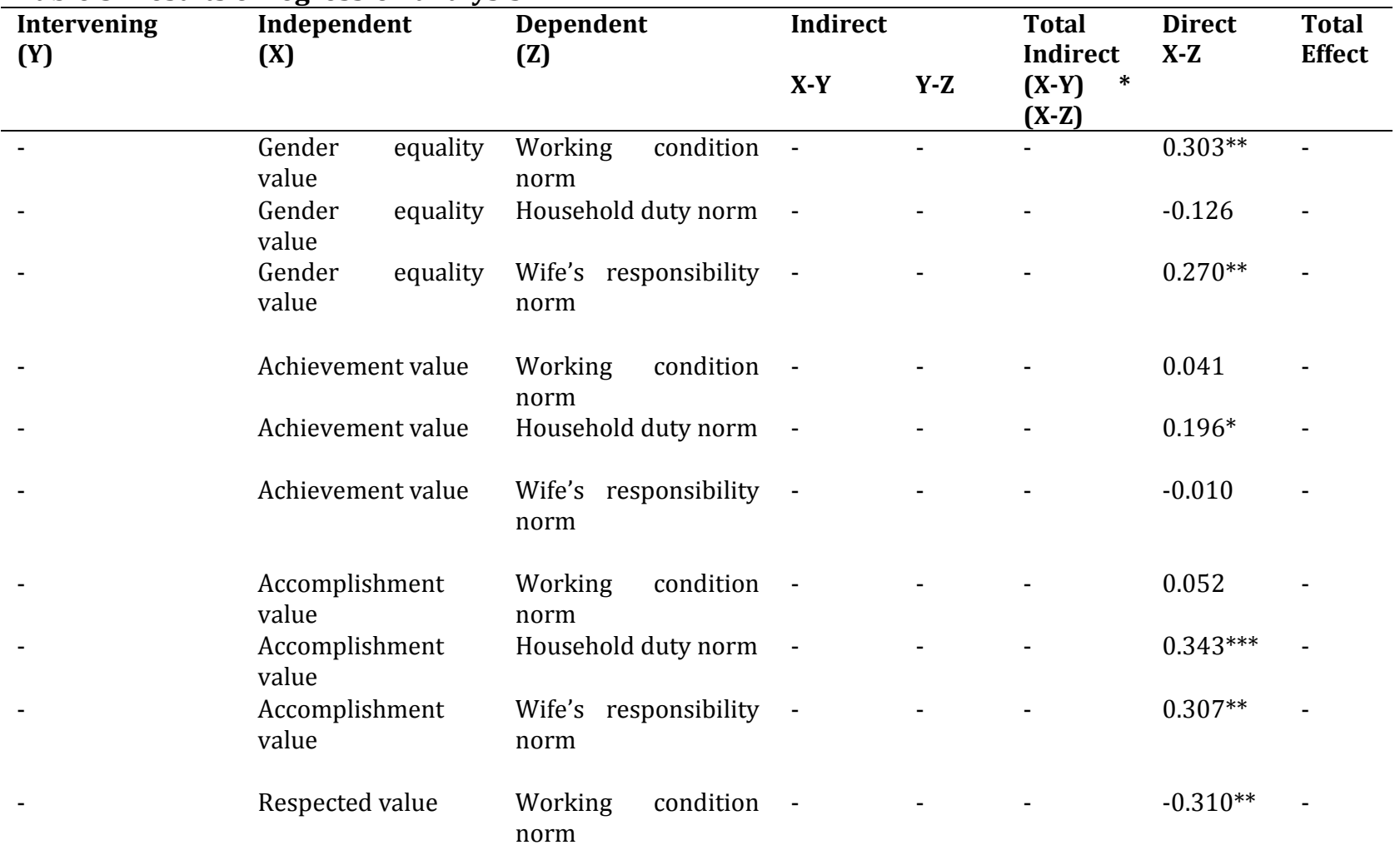




\begin{tabular}{|c|c|c|c|c|c|c|c|c|}
\hline - & Respected value & \multicolumn{2}{|c|}{ Household duty norm } & - & - & - & 0.003 & - \\
\hline - & Respected value & \multicolumn{2}{|c|}{$\begin{array}{l}\text { Wife's responsibility } \\
\text { norm }\end{array}$} & - & - & - & $0.200^{*}$ & - \\
\hline - & $\begin{array}{l}\text { Working condition } \\
\text { norm }\end{array}$ & \multicolumn{2}{|l|}{ Wife's respect } & - & - & - & 0.153 & - \\
\hline - & $\begin{array}{l}\text { Working condition } \\
\text { norm }\end{array}$ & $\begin{array}{l}\text { Maintain } \mathrm{h} \\
\text { prestige }\end{array}$ & husband's & - & - & - & -0.051 & - \\
\hline - & $\begin{array}{l}\text { Household duty } \\
\text { norm }\end{array}$ & \multicolumn{2}{|c|}{ Wife's respect } & - & - & - & $0.342^{* * *}$ & - \\
\hline - & $\begin{array}{l}\text { Household duty } \\
\text { norm }\end{array}$ & $\begin{array}{l}\text { Maintain } \mathrm{h} \\
\text { prestige }\end{array}$ & husband's & - & - & - & 0.012 & - \\
\hline - & $\begin{array}{l}\text { Wife's } \\
\text { responsibility norm }\end{array}$ & \multicolumn{2}{|l|}{ Wife's respect } & - & - & - & $0.652^{* * *}$ & - \\
\hline - & $\begin{array}{l}\text { Wife's } \\
\text { responsibility norm }\end{array}$ & $\begin{array}{l}\text { Maintain } h \mathrm{l} \\
\text { prestige }\end{array}$ & husband's & - & - & - & $0.412^{* * *}$ & - \\
\hline - & Wife's respect & $\begin{array}{l}\text { Usage of } \\
\text { income }\end{array}$ & wife's & - & - & - & 0.109 & - \\
\hline - & $\begin{array}{l}\text { Maintain husband's } \\
\text { prestige }\end{array}$ & $\begin{array}{l}\text { Usage of } \\
\text { income }\end{array}$ & wife's & - & - & - & $0.415^{* * *}$ & - \\
\hline - & $\begin{array}{l}\text { Gender equality } \\
\text { value }\end{array}$ & $\begin{array}{l}\text { Usage of } \\
\text { income }\end{array}$ & wife's & - & - & - & -0.083 & - \\
\hline - & Achievement value & $\begin{array}{l}\text { Usage of } \\
\text { income }\end{array}$ & wife's & - & - & - & 0.146 & - \\
\hline- & $\begin{array}{l}\text { Accomplishment } \\
\text { value }\end{array}$ & $\begin{array}{l}\text { Usage of } \\
\text { income }\end{array}$ & wife's & - & - & - & $0.225^{* *}$ & - \\
\hline- & Respected value & $\begin{array}{l}\text { Usage of } \\
\text { income }\end{array}$ & wife's & - & - & - & -0.055 & - \\
\hline
\end{tabular}

Table 5: Results of regression analysis (continued)

\begin{tabular}{|c|c|c|c|c|c|c|c|}
\hline $\begin{array}{l}\text { Intervening } \\
(\mathrm{Y})\end{array}$ & $\begin{array}{l}\text { Independent } \\
(\mathrm{X})\end{array}$ & $\begin{array}{l}\text { Dependent } \\
\text { (Z) }\end{array}$ & $\begin{array}{l}\text { Indirect } \\
\mathrm{X}-\mathrm{Y}\end{array}$ & $\mathbf{Y}-\mathbf{Z}$ & $\begin{array}{l}\text { Total } \\
\text { Indirect } \\
(\mathrm{X}-\mathrm{Y}) *(\mathrm{X}-\mathrm{Z})\end{array}$ & $\begin{array}{l}\text { Direct X- } \\
\mathrm{Z}\end{array}$ & $\begin{array}{l}\text { Total } \\
\text { Effect }\end{array}$ \\
\hline- & $\begin{array}{l}\text { Gender equality } \\
\text { value }\end{array}$ & Wife's respect & - & - & - & $0.329 *$ & - \\
\hline- & $\begin{array}{l}\text { Gender equality } \\
\text { value }\end{array}$ & $\begin{array}{l}\text { Maintain } \\
\text { husband's } \\
\text { prestige }\end{array}$ & - & - & - & -0.101 & - \\
\hline- & $\begin{array}{l}\text { Achievement } \\
\text { value }\end{array}$ & Wife's respect & - & - & - & 0.153 & - \\
\hline- & $\begin{array}{l}\text { Achievement } \\
\text { value }\end{array}$ & $\begin{array}{l}\text { Maintain } \\
\text { husband's } \\
\text { prestige }\end{array}$ & - & - & - & 0.100 & - \\
\hline- & $\begin{array}{l}\text { Accomplishment } \\
\text { value }\end{array}$ & Wife's respect & - & - & - & $0.387^{* * *}$ & - \\
\hline- & $\begin{array}{l}\text { Accomplishment } \\
\text { value }\end{array}$ & $\begin{array}{l}\text { Maintain } \\
\text { husband's } \\
\text { prestige }\end{array}$ & - & - & - & 0.120 & - \\
\hline- & Respected value & Wife's respect & - & - & - & -0.011 & - \\
\hline- & Respected value & $\begin{array}{l}\text { Maintain } \\
\text { husband's } \\
\text { prestige }\end{array}$ & - & - & - & $0.250^{*}$ & - \\
\hline $\begin{array}{l}\text { Working } \\
\text { condition }\end{array}$ & $\begin{array}{l}\text { Gender equality } \\
\text { value }\end{array}$ & Wife's respect & $0.303^{* *}$ & 0.153 & Not sig. & $0.329 *$ & - \\
\hline
\end{tabular}




\begin{tabular}{|c|c|c|c|c|c|c|c|}
\hline $\begin{array}{l}\text { Working } \\
\text { condition } \\
\text { norm }\end{array}$ & $\begin{array}{l}\text { Gender equality } \\
\text { value }\end{array}$ & $\begin{array}{l}\text { Maintain } \\
\text { husband's } \\
\text { prestige }\end{array}$ & $0.303^{* *}$ & -0.051 & Not sig. & -0.101 & - \\
\hline $\begin{array}{l}\text { Working } \\
\text { condition } \\
\text { norm }\end{array}$ & $\begin{array}{l}\text { Achievement } \\
\text { value }\end{array}$ & Wife's respect & 0.041 & 0.153 & Not sig & 0.153 & - \\
\hline $\begin{array}{l}\text { Working } \\
\text { condition } \\
\text { norm }\end{array}$ & $\begin{array}{l}\text { Achievement } \\
\text { value }\end{array}$ & $\begin{array}{l}\text { Maintain } \\
\text { husband's } \\
\text { prestige }\end{array}$ & 0.041 & -0.051 & Not sig & 0.100 & - \\
\hline $\begin{array}{l}\text { Working } \\
\text { condition } \\
\text { norm }\end{array}$ & $\begin{array}{l}\text { Accomplishment } \\
\text { value }\end{array}$ & Wife's respect & 0.052 & 0.153 & Not sig & $0.387^{* * *}$ & - \\
\hline $\begin{array}{l}\text { Working } \\
\text { condition } \\
\text { norm }\end{array}$ & $\begin{array}{l}\text { Accomplishment } \\
\text { value }\end{array}$ & $\begin{array}{l}\text { Maintain } \\
\text { husband's } \\
\text { prestige }\end{array}$ & 0.052 & -0.051 & Not sig & 0.120 & - \\
\hline $\begin{array}{l}\text { Working } \\
\text { condition } \\
\text { norm }\end{array}$ & Respected value & Wife's respect & $-0.310^{* *}$ & 0.153 & Not sig & $0.387^{* * *}$ & - \\
\hline $\begin{array}{l}\text { Working } \\
\text { condition } \\
\text { norm }\end{array}$ & Respected value & $\begin{array}{l}\text { Maintain } \\
\text { husband's } \\
\text { prestige }\end{array}$ & $-0.310^{* *}$ & -0.051 & Not sig & 0.100 & - \\
\hline $\begin{array}{l}\text { Household } \\
\text { duty norm }\end{array}$ & $\begin{array}{l}\text { Gender equality } \\
\text { value }\end{array}$ & Wife's respect & -0.126 & $0.342^{* * *}$ & Not sig & $0.329 *$ & - \\
\hline $\begin{array}{l}\text { Household } \\
\text { duty norm }\end{array}$ & $\begin{array}{l}\text { Achievement } \\
\text { value }\end{array}$ & Wife's respect & $0.196^{*}$ & $0.342^{* * *}$ & $0.067032^{*}$ & 0.153 & - \\
\hline $\begin{array}{l}\text { Household } \\
\text { duty norm }\end{array}$ & $\begin{array}{l}\text { Accomplishment } \\
\text { value }\end{array}$ & Wife's respect & $0.343^{* * *}$ & $0.342^{* * *}$ & $0.117306^{* * *}$ & $0.387^{* * *}$ & 0.504306 \\
\hline $\begin{array}{l}\text { Household } \\
\text { duty norm }\end{array}$ & Respected value & Wife's respect & 0.003 & $0.342^{* * *}$ & Not sig & $0.387^{* * *}$ & - \\
\hline $\begin{array}{l}\text { Household } \\
\text { duty norm }\end{array}$ & $\begin{array}{l}\text { Gender equality } \\
\text { value }\end{array}$ & $\begin{array}{l}\text { Maintain } \\
\text { husband's } \\
\text { prestige }\end{array}$ & -0.126 & 0.012 & Not sig & -0.101 & - \\
\hline $\begin{array}{l}\text { Household } \\
\text { duty norm }\end{array}$ & $\begin{array}{l}\text { Achievement } \\
\text { value }\end{array}$ & $\begin{array}{l}\text { Maintain } \\
\text { husband's } \\
\text { prestige }\end{array}$ & $0.196^{*}$ & 0.012 & Not sig & 0.100 & - \\
\hline $\begin{array}{l}\text { Household } \\
\text { duty norm }\end{array}$ & $\begin{array}{l}\text { Accomplishment } \\
\text { value }\end{array}$ & $\begin{array}{l}\text { Maintain } \\
\text { husband's } \\
\text { prestige }\end{array}$ & $0.343^{* * *}$ & 0.012 & Not sig & 0.120 & - \\
\hline $\begin{array}{l}\text { Household } \\
\text { duty norm }\end{array}$ & Respected value & $\begin{array}{l}\text { Maintain } \\
\text { husband's } \\
\text { prestige }\end{array}$ & 0.003 & 0.012 & Not sig & 0.100 & - \\
\hline $\begin{array}{l}\text { Wife's } \\
\text { responsibility } \\
\text { norm }\end{array}$ & $\begin{array}{l}\text { Gender equality } \\
\text { value }\end{array}$ & Wife's respect & $0.270^{* *}$ & $0.652^{* * *}$ & $0.17604^{* *}$ & $0.329 *$ & 0.50504 \\
\hline $\begin{array}{l}\text { Wife's } \\
\text { responsibility } \\
\text { norm }\end{array}$ & $\begin{array}{l}\text { Achievement } \\
\text { value }\end{array}$ & Wife's respect & -0.010 & $0.652^{* * *}$ & Not sig & $0.652^{* * *}$ & - \\
\hline $\begin{array}{l}\text { Wife's } \\
\text { responsibility } \\
\text { norm }\end{array}$ & $\begin{array}{l}\text { Accomplishment } \\
\text { value }\end{array}$ & Wife's respect & $0.307^{* *}$ & $0.652^{* * *}$ & $0.200164^{* *}$ & $0.387^{* * *}$ & 0.587164 \\
\hline $\begin{array}{l}\text { Wife's } \\
\text { responsibility } \\
\text { norm }\end{array}$ & Respected value & Wife's respect & $0.200^{*}$ & $0.652^{* * *}$ & $0.1304^{*}$ & -0.011 & - \\
\hline $\begin{array}{l}\text { Wife's } \\
\text { responsibility } \\
\text { norm }\end{array}$ & $\begin{array}{l}\text { Gender equality } \\
\text { value }\end{array}$ & $\begin{array}{l}\text { Maintain } \\
\text { husband's } \\
\text { prestige }\end{array}$ & $0.270^{* *}$ & $0.412^{* * *}$ & $0.11124^{* *}$ & -0.101 & - \\
\hline
\end{tabular}




\begin{tabular}{|c|c|c|c|c|c|c|c|}
\hline $\begin{array}{l}\text { Wife's } \\
\text { responsibility } \\
\text { norm }\end{array}$ & $\begin{array}{l}\text { Achievement } \\
\text { value }\end{array}$ & $\begin{array}{l}\text { Maintain } \\
\text { husband's } \\
\text { prestige }\end{array}$ & -0.010 & $0.412^{* * *}$ & Not sig. & 0.100 & - \\
\hline $\begin{array}{l}\text { Wife's } \\
\text { responsibility } \\
\text { norm }\end{array}$ & $\begin{array}{l}\text { Accomplishment } \\
\text { value }\end{array}$ & $\begin{array}{l}\text { Maintain } \\
\text { husband's } \\
\text { prestige }\end{array}$ & $0.307^{* *}$ & $0.412^{* * *}$ & $0.126484^{* *}$ & 0.120 & - \\
\hline $\begin{array}{l}\text { Wife's } \\
\text { responsibility } \\
\text { norm }\end{array}$ & Respected value & $\begin{array}{l}\text { Maintain } \\
\text { husband's } \\
\text { prestige }\end{array}$ & $0.200^{*}$ & $0.412^{* * *}$ & $0.0824^{*}$ & $0.250^{*}$ & 0.3324 \\
\hline
\end{tabular}

\section{Hypotheses Testing}

Husband's Personal Value: From four factors constructing husband's personal value, only three factors significantly influence endogen variable norm and perception: gender equality value, accomplishment value, and respected value. The result shows that gender equality value significantly influence wife's responsibility norm with a beta coefficients of $0.270^{* *}$. Gender equality value measures the degree of importance husbands put in values such as warm, self-respect, universalism, and benevolence. Benevolence value (preservation and enhancement of the welfare of people with whom one is in frequent personal contact) has the biggest factor loading. Based on the definition, we can infer that people with high benevolence value will give more attention to the people he or she is close to. Meanwhile wife's responsibility norm is husband's norm about wife's responsibility consist of child education and respect to husband as head of household. In this case, a husband with high benevolence value will give more attention to his family. This might be the explanation why gender equality value will significantly influence husband's wife's responsibility norm. Accomplishment value will have direct influence on using wife's income for family expenditure variable with a beta coefficient value of $0.225^{* * *}$. Accomplishment value means to succeed at what you want to do. The higher importance a husband gives to this value the higher usage of wife's income for family expenditure. Meanwhile husband's respected value have direct, significant influence on both wife's responsibility norm with beta coefficient value of $0.200^{*}$ and maintain husband's prestige with beta coefficient value of $0.250^{*}$. Respected value means to be admired by others and to receive recognition. It is apparent that the higher this value, the more importance husband gives on how well his wife will be able to maintain his prestige in front of his child and public and how his working wife will still respect him as the head of the household.

Husband's Norm: From three factors constructing "husband's norm about wife working outside home" variable, only household duty norm and wife's responsibility norm influence perception significantly. Household duty norm significantly influences husband's perception towards wife's respect with a beta coefficient value of $0.342^{* * *}$. Meanwhile wife's responsibility norm affects husband's perception towards wife's respect with a beta coefficient value of $0.652^{* * *}$. Wife's responsibility norm also has significant effect on husband's perception towards how well wife maintain husband's prestige with a beta coefficient value of $0.412^{* * *}$. Household duty norm measures husband's norm about wife's household duty. All the factor loadings of items constructing this factor are all negative. This means that husband who has working wife still see child caring (hand-feed the child, bathe the child), home cleaning, and cooking as an important household duty, but it is no longer necessarily performed by wife. This might explain the phenomena of usage of helper in a lot of modern household in Jakarta and Bandung to do those household duties. Wife's responsibility norm measures husband's norm about wife's responsibility. It is very interesting to see that even though husbands did not see child caring as wife's responsibility anymore, they still see child education (teach manners, values, politeness, etc.) as an important responsibility of wives.

Husband's Perception: Husband's perception constructed by two factors: wife's respect and maintain husband's prestige. From these two factors, only maintain husband's prestige has significant influence on dependent variable "using wife income for family expenditure" with a beta coefficient value of $0.415^{* * *}$. Maintain husband's prestige measures husband's perception towards how well wife maintain husband's prestige in front of public and in front of his children. We can infer that if husband sees his wife is able to maintain his prestige in front of his child and public he will not be reluctant to use wife's income for family expenditure. 
Model Testing: At the beginning of the study, we try to propose a model to find out the correlation between husband's norm, value, and perception towards his working wife and usage of wife's income for family expenditure (Figure 1). We conducted regression analysis to examine the relation between variables. As can be seen in table 5, not all variables have significant impact to usage of wife's income for family expenditure. Therefore, we proposed a new model, which picture how husband's norm, value, and perception influence usage of wife's income for family expenditure in a family. This model can be seen in Figure 2.

\section{Figure 2: Proposed Model}

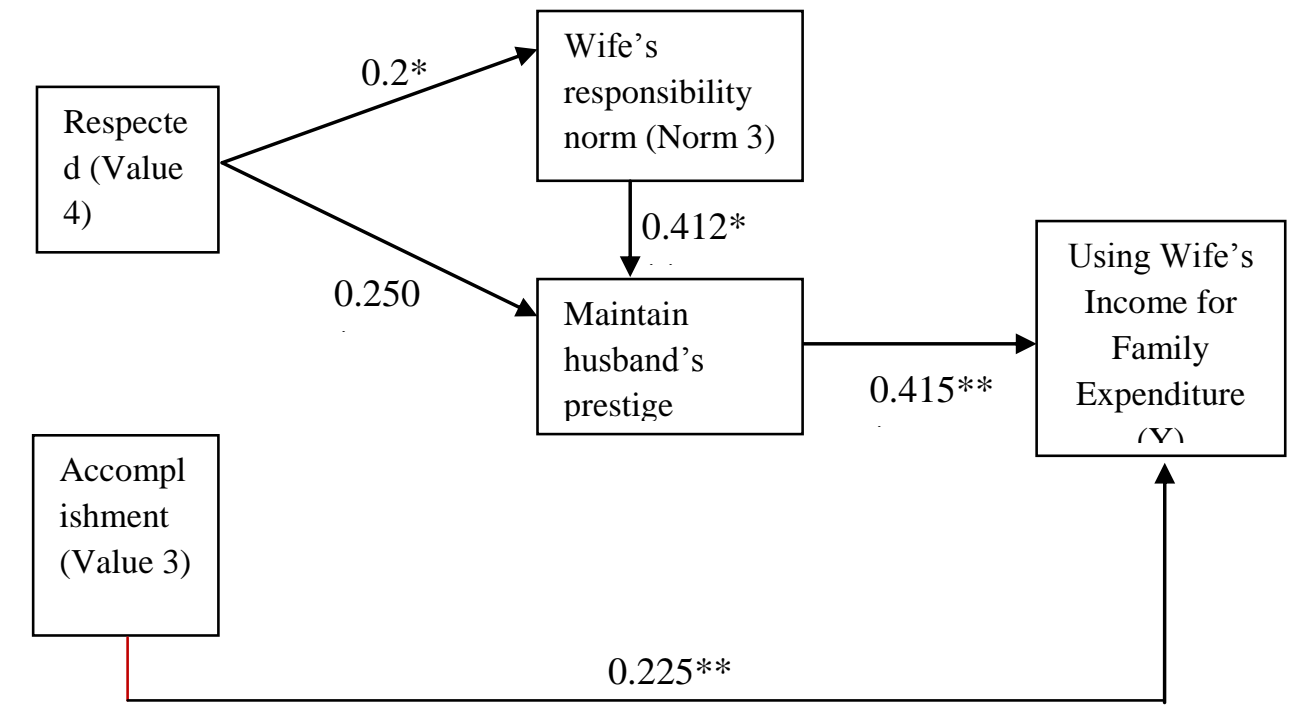

Our findings support some of the hypotheses (see table 6).

Table 6: Summary result of hypotheses

\begin{tabular}{|c|c|c|}
\hline Variables & Hypotheses & Test Result \\
\hline Husband's value affects norm & H1a & $\begin{array}{l}\text { Supported } \\
\text { partially }\end{array}$ \\
\hline Husband's value affects perception & $\mathrm{H} 1 \mathrm{~b}$ & $\begin{array}{l}\text { Supported } \\
\text { partially }\end{array}$ \\
\hline Husband's value affects usage of wife's income for family expenditure & $\mathrm{H} 1 \mathrm{c}$ & $\begin{array}{l}\text { Supported } \\
\text { partially }\end{array}$ \\
\hline Husband's norm affects husband's perception & $\mathrm{H} 2$ & $\begin{array}{l}\text { Supported } \\
\text { partially }\end{array}$ \\
\hline $\begin{array}{l}\text { Husband's perception affects usage of wife's income for family } \\
\text { expenditure }\end{array}$ & H3 & $\begin{array}{l}\text { Supported } \\
\text { partially }\end{array}$ \\
\hline
\end{tabular}

Theoretical and Managerial Implication: This study give contribution in understanding the way husband's norm, perception, and value affect the usage of wife's income for family expenditure. This study also shows values and norms of Indonesian husbands, particularly husband who lives in big cities such as Jakarta and Bandung. This research also shows perception of husband towards working women, especially husband's perception towards his working wife. The research findings stated that husbands nowadays no longer think that using wife's income for family expenditure as something that is inappropriate. The statement is 
especially true for husbands who belief in respected and accomplishment value. Husbands who have respected value (needs to be admired by others and to receive recognition) significantly influence husband's belief about wife's responsibility norm and husband's perception about how well his wife maintains husband's prestige. Husband's perception about how well his wife maintain his prestige significantly influence husband's willingness to use wife's income for family expenditure. Finding shows about how working wife should behave in her family and in public without making her husband feels emasculated. These findings are especially useful for government and marketers in patriarchal countries such as Indonesia and many other Asian countries where husbands still think that he is the head of the household.

Findings on the importance husbands put in how well their wife can maintain their prestige in front of public and in front of their children give an insight on how marketers should tailor their communication strategy, so that husbands will not feel emasculated when they have to use their spouse's income in buying those goods or services. The findings also important for government for example if the government wants to create a public campaign promoting gender equality.

Limitation and Future Research: The limitation of the research is the sampling method, which is convenience sampling so the findings will not be able to be generalized throughout Indonesia. The study needs to be replicated with larger number of sample and diversified geographical regions so the result can be generalized to cover Indonesia area.

\section{References}

Blood, R. D. \& Wolfe, D. M. (1960). Husbands and Wives, The Free Press, New York, 1960.

Fehr, E. \& Fischbacher, U. (2004). Social Norms and Human Cooperation. TRENDS in Cognitive Sciences, 8 (4).

Filiatrault, P. \& Brent-Ritchie, J. R. (1980). Joint Purchasing Decisions: A Comparison of Influence Structure in Family and Couple Decision Making Units. Journal of Consumer Research, 7(2), 131-140.

Kandori, M. (1992). Social Norms and Community Enforcement. Review of Economic Studies, 59, 63-80

Gianopulos, A. \& Mitchell, H. E. (1957). Marital Disagreement in Working Wife Marriages as A Function of Husband's Attitude Towards Wife's Employment. Marriage and Family Living, 19(4), 373-378.

Harcar, T., Spillan, J. E. \& Orsay, K. (2005). A Multi-National Study of Family Decision-making. Multinational Business Review, 13(2), 3

Hair, J. F., Black, W. C., Babin, B. J. \& Anderson, R. E. (2010). Multivariate Data Analysis, Pearson Prentice Hall

Kotler, P. (2002). Marketing Management, 11th edition. United States: Prentice Hall.

Lapinski, M. K. \& Rimal, R. N. (2005). An Explication of Social Norms, International Communication Association, 127-147

Lee-Christina K. C. \& Beatty, S. E. (2002). Family structure and influence in family decision-making. Journal of Consumer Marketing, 19(1), $24-41$

Makgosa, R. (2007). Exploring the Impact of Ethnicity on Conflict Resolution in Joint Purchase Decision Making. Journal of American Academy of Business, Cambridge, 11(2), 205

Ndubisi, N. 0. \& Koo, J. (2006). Family structure and joint purchase decisions: two products analysis. Management Research News, 29(1/2), 53

Pallant, J. (2011). Survival Manual Astep by Step Guide to Data Analysis Using SPSS, $4^{\text {th }}$ ed.Allen \&Uwin: Crows Nest

Qualls, W. J. (1987). Household Decision Behavior: The Impact of Husbands' and Wives' Sex Role Orientation. Journal of Consumer Research, 14(2), 264-279.

Rokeach, M. (1973). The Nature of Human Values, Free Press, New York, NY

Rosen, D. L. \& Granbois, D. H. (1983). Determinant of Role Structure in Family Financial Management. Journal of Consumer Research, 10(2), 253-258.

Scanzoni, J. (1977). Changing Sex Roles and Emerging Directions in Family Decision Making. Journal of Consumer Research, 4(3), 185-188.

Schaninger, C. M., Buss, W. C. \& Grover, R. (1982). The Effect of Sex Roles on Family Economic Handling and Decision Influence, In an Assessment of Marketing Thought and Practice, B. Walker, ed., American Marketing Association, Chicago. 
So, S. L. M. \& Yau, O. H. M. (2006). Chinese Husbands: A Path-Analytic Study of a Model of Family Buying Decisions. Asia Pasific Management Review, 11(5), 283-294.

Spiro, R. (1983). Persuasion in Family Decision Making. Journal of Consumer Research, 9(4), 393-402.

Stafford, M. R., Ganesh, G. \& Lucket, M. G. (2011). Perceived Spousal Influence in the Service Decision Making Process: A Cross Cultural Investigation. Journal of Applied Business Research, 12(4).

Vanvara, B. (2009). The influence of personal values on environmental attitude, product aesthetics, and product evaluation, ProQuest Dissertations and Theses; ABI/INFORM Complete

Xia, Y., Zafar, U. A., Morry, G., Hwa, N. K., Li, T. W. \& Ying, W. T. C. (2006). Spousal influence in Singaporean family purchase decision-making process: A cross-cultural comparison. Asia Pacific Journal of Marketing and Logistics, 18(3), 201 - 222 